Características antioxidantes, fisicoquímicas y microbiológicas de jugo fermentado y sin fermentar de tres variedades de pitahaya (Hylocereus spp)

\title{
Antioxidant, physicochemical and microbiological characteristics of fermented and unfermented drink of three varieties of dragon fruits (Hylocereus spp)
}

\author{
Ochoa-Velasco Carlos Enrique ${ }^{1, *}$; García-Vidal Verónica'; Luna-Guevara Juan José ${ }^{1}$; \\ Luna-Guevara María Lorena ${ }^{1}$; Hernández-Carranza Paola ${ }^{1}$; Guerrero-Beltrán José Ángel ${ }^{2}$ \\ Facultad de Ingeniería Química, Benemérita Universidad Autónoma de Puebla, México. \\ 2 Departamento de Ingeniería Química, Alimentos y Ambiental, Universidad de las Américas Puebla, México.
}

Recibido 05 noviembre 2012; aceptado 16 diciembre 2012.

\begin{abstract}
Resumen
Al jugo de pitahaya de tres variedades (roja, rosa y blanca) se le evaluó el pH, sólidos solubles totales, acidez titulable, color, compuestos fenólicos, capacidad antioxidante, mientras que al jugo de pitahaya fermentado con Saccharomyces cerevisiae se le evaluaron además el número de bacterias mesófilas aerobias, mohos y levaduras y contenido alcohólico. Los sólidos solubles totales y pH disminuyeron en el jugo fermentado en todas las variedades de pitahaya. El contenido de compuestos fenólicos fue mayor en el jugo de pitahaya roja, seguido de la rosa y la blanca con valores promedios de 45,3, 32,1y 24,6 mg de ácido gálico/100 mL de jugo, respectivamente. Por ende, la capacidad antioxidante fue superior en el jugo de pitahaya roja (160,84 mg de Trolox/100 mL de jugo). La fermentación del jugo de pitahaya incrementó el contenido de compuestos fenólicos (variando de 45,31 a 51,38 mg de ácido gálico/100 mL de jugo, en pitahaya roja), aunque disminuyó la capacidad antioxidante (variando de 124,51 a $82,96 \mathrm{mg}$ de Trolox/100 mL de jugo, en pitahaya rosa). El contenido de alcohol de los jugos fermentados fue entre 2,9 y 3,9\% (v/v). Al final de la fermentación la carga máxima de mohos y levaduras $(1580 \mathrm{UFC} / \mathrm{mL})$ la presentó el jugo fermentado de pitahaya blanca. Sin embargo, después de la pasteurización el crecimiento microbiano en todos los tratamientos fue menor a 10 UFC/mL. La elaboración de subproductos como el jugo fermentado es una alternativa viable para la comercialización de productos derivados de la pitahaya.
\end{abstract}

Palabras clave: Pitahaya, compuestos fenólicos, capacidad antioxidante, jugo fermentado.

\begin{abstract}
Dragon fruit juice from three variety (red, pink, and white) were evaluated in $\mathrm{pH}$, total soluble solids, titratable acidity, color, phenolic compounds, antioxidant capacity, while in fermented dragon fruit juice with Saccharomyces cerevisiae were also evaluated the mesophylls and yeasts plus molds, and alcohol grade. The contents of total soluble solids and $\mathrm{pH}$ decreased in the fermented juice of all varieties as consequence of the fermentation process. The phenolic compounds were higher in the red dragon fruit juice, followed by the pink and white dragon fruit with average values of 45.3, 32.1 y $24.6 \mathrm{mg}$ of Gallic acid/100 mL of juice, respectively. Therefore, the antioxidant activity was higher in the red dragon fruit juice (160.84 mg of Trolox/100 mL of juice). Fermenting the juice increased the phenolic compounds (changing of 45.31 to 51.38 $\mathrm{mg}$ of galic acid/100 mL of juice, in red dragon fruit), however decreased the antioxidant activity (124.51 to $82.96 \mathrm{mg}$ of Trolox $/ 100 \mathrm{~mL}$ of juice, in pink dragon fruit). The alcohol grades in the fermented juices were among 2.9 to $3.9 \%(\mathrm{v} / \mathrm{v})$. At the end of the fermentation the higher yeasts plus molds $(1580 \mathrm{CFU} / \mathrm{mL})$ were in the white dragon fruit juice. However, after the pasteurization process the microbial load in all treatments were lower to $10 \mathrm{CFU} / \mathrm{mL}$. The development of products such as fermented juice is a viable alternative for the commercialization of products derived from dragon fruit.
\end{abstract}

Keywords: Dragon fruit, phenolic compounds, antioxidant capacity, fermented juice.

\footnotetext{
* Autor para correspondencia

Email: carlosenriqueov@ hotmail.com (C. Ochoa)
} 


\section{Introducción}

La pitahaya (Hylocereus spp) es una fruta originaria de América perteneciente a la familia de las cactáceas, subfamilia Cactoideae, Tribu Hylocereae y género Hylocereus (Raveh et al., 1993); existen diferentes especies de pitahaya; sin embargo, la especie Hylocereus undatus es la más cultivada a nivel mundial con un área de cultivo del 71,5\% (Betancourt et al., 2010) y la más apreciada comercialmente debido a que sus frutos son comercializados fácilmente en mercados nacionales e internacionales (Legaria et al., 2005). La pitahaya es una fruta de sabor dulce y de diferentes coloraciones, con un peso de hasta $700 \mathrm{~g}$ y un largo y diámetro de 15 y $10 \mathrm{~cm}$, respectivamente. La piel presenta coloraciones roja-rosácea, sin espinas, pero con escamas a manera de alas que contribuyen a la atractiva apariencia del fruto (Corrales-García y Canche-Canche, 2008; Wybraniec y Mizrahi, 2002). Es una fruta muy apreciada debido a que es considerada un fruto exótico en los mercados europeos y asiáticos (Castillo, 2006); mientras que en Estados Unidos y Europa demandan pulpa de pitahaya como ingrediente en alimentos o bien como colorante natural (Esquivel y ArayaQuesada, 2012). México produce alrededor de 2155,3 toneladas al año y es el tercer productor mundial de pitahaya, únicamente por debajo de Colombia y Nicaragua (SIAP, 2011). Puebla alcanza una producción de 57,25 toneladas en aproximadamente 17 hectáreas sembradas (SIAP, 2011). Sin embargo, gran parte de la producción se pierde debido a la escasa tecnología pre y poscosecha que se aplica en la producción de pitahaya tanto en fresco como procesado. Una posible forma de comercializar la pitahaya es a partir de la obtención de jugo fermentado o "vino de pitahaya", siendo ésta una alternativa viable para que los productores de pitahaya puedan darle valor agregado a sus productos, abriendo un nuevo mercado potencialmente económico.
La palabra vino se refiere al producto obtenido de la fermentación del jugo de uva (Brown et al., 1989). Mientras que el vino de frutas es la bebida alcohólica obtenida por la parcial o completa fermentación de jugos de frutas fresco, concentrado o reconstituido; o macerado de pulpa con la adición de agua, azúcar o miel (Ferreyra et al., 2009).

Por lo anterior, el objetivo de esta investigación fue elaborar una bebida fermentada (vino) a partir de jugo de diferentes variedades de pitahaya (Hylocereus spp).

\section{Material y Métodos}

\subsection{Materia prima}

Se utilizaron tres variedades de pitahaya (Hylocereus spp) de diferentes coloraciones de pulpa, roja, rosa y blanca obtenidos en el mercado de Huixcolotla, Puebla, México. Las frutas fueron seleccionadas con una coloración uniforme, sin daños físicos $\mathrm{y}$ microbiológicos visibles (Figura 1).

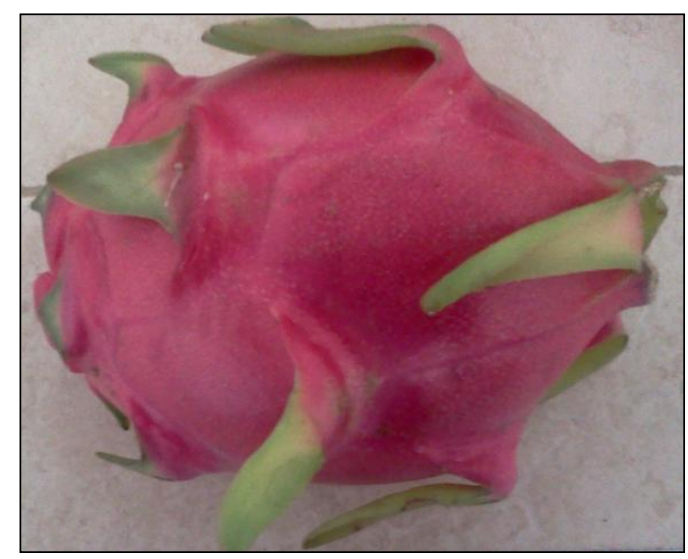

Figura 1. Pitahaya (Hylocereus spp).

\subsection{Características físicas}

Se realizó la caracterización física de la pitahaya evaluando la dimensión (largo y ancho) y el peso (peso de la pulpa y peso de la cáscara) con la ayuda de un vernier y una balanza analítica (Ohaus modelo Scout Pro, USA), respectivamente. 


\subsection{Elaboración de jugo y fermentación}

Se obtuvo el jugo con pulpa con la ayuda de un procesador Black and Dekker. Posteriormente, el jugo fue filtrado 2 veces con la utilización de un tamiz $(0,297 \mathrm{~mm})$ para remover los materiales suspendidos. El contenido de sólidos solubles totales del jugo se ajusto (sacarosa) a $15 \%$ y se les agregó Saccharomyces cerevisiae $(1 \mathrm{~mL} / \mathrm{L}$ de jugo) obtenida del cepario de la UDLAP. El jugo (mosto) se almacenó en recipientes plásticos (PET) y se dejó fermentar en la oscuridad durante un mes a temperatura ambiente $\left(20-25{ }^{\circ} \mathrm{C}\right)$. El jugo fermentado se tamizó y se centrifugó a $12000 \mathrm{rpm}$ durante 40 minutos para obtener la bebida fermentada clarificada.

\subsection{Pasteurización}

La mitad de los tres jugos fermentados obtenidos se pasteurizó a $70{ }^{\circ} \mathrm{C}$ durante 3 minutos con la finalidad de inactivar la levadura y así evitar continuar con la fermentación del jugo. El jugo fermentado y pasteurizado se envaso en recipientes de plástico estériles y se mantuvo a temperatura ambiente y en oscuridad hasta su análisis.

\subsection{Características fisicoquímicas}

Se determinó el $\mathrm{pH}$, los sólidos solubles totales y la acidez titulable en jugo fresco y fermentado de pitahaya siguiendo los métodos $981,12,932,12$ y 942,15 de la AOAC (2000), respectivamente.

\subsection{Color}

Se colocaron $10 \mathrm{~mL}$ de jugo freso o fermentado en un caja Petri de $6 \mathrm{~cm}$ de diámetro y $1,5 \mathrm{~cm}$ de profundidad. Se evaluaron los parámetros $L$ (Luminosidad, blanco-negro), $\quad a$ (verde-rojo), y $b$ (amarillo-azul) de la escala de Hunter, utilizando un colorímetro Colorgard System/05 (BYK-Gardner GmbH, Geretsried, Alemania) en el modo transmitancia.

\subsection{Compuestos fenólicos}

Se realizó siguiendo el método propuesto por Gao et al. (2000) con modificaciones. En un tubo ámbar se mezcló $2 \mathrm{~mL}$ de agua destilada con $200 \mu \mathrm{L}$ de reactivo de FolinCiocalteau y $100 \mu \mathrm{L}$ de muestra. Esta mezcla se incubó durante 3 minutos a 24$26{ }^{\circ} \mathrm{C}$. Posteriormente se añadió $1 \mathrm{~mL}$ de solución de $\mathrm{Na}_{2} \mathrm{CO}_{3}$ al $20 \%$, se mezcló perfectamente y se incubó durante 1 hora en oscuridad a $24-26{ }^{\circ} \mathrm{C}$. La reacción se midió a una longitud de onda de $765 \mathrm{~nm}$ en un espectrofotómetro UV-Visible marca UNICO $®$, modelo $2800 \mathrm{H}$ (United Products \& Instruments, Inc., Dayton, NJ, USA) y se calculó el contenido de compuestos fenólicos (Ec. 1) utilizando una curva estándar de ácido gálico (33 mg de ácido gálico/ $100 \mathrm{~mL}$ con agua destilada). Los resultados se expresan en mg de ácido gálico por $100 \mathrm{~mL}$ de jugo fresco o fermentado de pitahaya.

$$
A G=\frac{(A-b)}{m}
$$

Donde $A G$ es el contenido de ácido gálico (mg de ácido gálico/100 mL), $A$ es la absorbancia de la muestra, $b$ es el intercepto (abs) y $m$ es la pendiente (abs/mg de ácido gálico/100 mL). La curva estándar fue $\mathrm{y}=4,108(\mathrm{x})-0,010\left(\mathrm{R}^{2}=\right.$ 0,989 ).

\subsection{Capacidad antioxidante}

Se determinó por $\mathrm{ABTS}^{+}$siguiendo la metodología propuesta por Kuskoski et al. (2004). El radical $\mathrm{ABTS}^{+}\left(2,2^{\prime}\right.$-azinobis (3ethylbenzthiozoline)-ácido 6-sulfonico) se obtuvo al mezclar 3,3 mg de persulfato de potasio y $19,4 \mathrm{mg}$ del reactivo de ABTS con $5 \mathrm{~mL}$ de agua destilada. La mezcla se agitó perfectamente y se dejó reposar en la oscuridad (16 horas) a $24-26^{\circ} \mathrm{C}$. Se realizó una mezcla del radical $\mathrm{ABTS}^{+}$con etanol absoluto, para obtener una absorbancia de $0,70 \pm 0,02$ a $754 \mathrm{~nm}$ en un espectrofotómetro UV-Visible. Se colocó $3920 \mu \mathrm{L}$ de la solución de radical ABTS:etanol absoluto a una celda de cuarzo y se registró la absorbancia inicial $\left(\mathrm{Abs}_{\text {inicial }}\right)$. Se adicionó $80 \mu \mathrm{L}$ de la muestra o extracto de la misma y 7 minutos después se registró la lectura de la absorbancia final $\left(\mathrm{Abs}_{\text {final }}\right)$. La capacidad antioxidante se calculó utilizando la ecuación 2 y 3 y para la cuantificación se utilizó una curva estándar de Trolox (0,2 mg de Trolox $/ \mathrm{mL})$. 
$\%$ Inhibición $=\frac{\left(A b s_{\text {inicial }}-A b s_{\text {final }}\right)}{A b s_{\text {inicial }}} * 100$

$$
A A=\frac{(\% \text { inhibición }-b)}{m} * 100
$$

Donde $A A$ es la capacidad antioxidante (mg T/100 mL de muestra), $b$ es el intercepto y $m$ es la pendiente (absorbancia/mg T/mL), la curva estándar fue $y=358,3(x)+0,819\left(R^{2}=0,98\right)$.

\subsection{Grado alcohólico}

El porcentaje de alcohol contenido en el jugo fermentado, se realizó por el método oficial de la unión europea (EurLex, 2004).

\subsection{Carga microbiana}

Las bacterias mesófilas aerobias (BMA) y mohos y levaduras ( $M$ y $L$ ) se cuantificaron en agar nutritivo (BD Bioxon, Cuautitlán Itzcalli, México) y agar papa dextrosa acidificado (10\% de ácido tartárico) (BD Bioxon, Cuautitlán Itzcalli, México), de acuerdo a la Normas Oficiales Mexicanas NOM-092-SSA1-1994 y la NOM-111-SSA1-1994, respectivamente. Las BMA se incubaron a $35 \pm 2{ }^{\circ} \mathrm{C}$ durante 2 días, y los $\mathrm{M}$ y $\mathrm{L}$ durante 5 días a $25 \pm 2$ ${ }^{\circ} \mathrm{C}$.

\subsection{Análisis estadístico}

Los resultados fueron realizados por triplicado y analizados mediante análisis de varianza utilizando el programa Minitab 14 (Minitab, Inc. PA, USA). Un valor de $p$ de 0,05 se utilizó para evaluar la existencia o no de diferencia significativa entre tratamientos de acuerdo a la prueba de Tukey.

\section{Resultados y discusión}

\subsection{Caracterización física de pitahaya producida en Puebla}

En la Tabla 1 se presenta la caracterización física de pitahayas producidas en la Mixteca Poblana. Los resultados obtenidos son similares a los reportados por Le et al. (2000). Ellos reportaron que las pitahayas presentan un peso total que oscila de $260 \mathrm{~g}$ hasta más de $500 \mathrm{~g}$, siendo las últimas las de mayor demanda en el mercado internacional. Esquivel et al. (2007a) reportaron que la variación en diámetros ecuatoriales varía desde 4,54 hasta $7,74 \mathrm{~cm}$ para la pitahaya cultivada en Costa Rica (Hylocereus costaricensis). Es importante recalcar que el tamaño y peso de los frutos se encuentra en función de la variedad o cultivar, utilización de buenas prácticas agrícolas, tipo de suelo y condiciones ambientales entre otros (Cerdas et al., 2006). Diferentes autores han concluido que las características de mayor relevancia en el fruto de pitahaya son el peso total, peso de pulpa, longitud, diámetro, grosor del pericarpio, sólidos solubles totales, número, longitud y anchura de brácteas (Castillo-Martínez et al., 2005).

Tabla 1

Caracterización física de pitahaya.

\begin{tabular}{lr}
\hline Características & Cantidades \\
\hline Longitud $(\mathrm{cm})$ & $12,12 \pm 1,3$ \\
Ancho $(\mathrm{cm})$ & $8,24 \pm 0,8$ \\
Peso total $(\mathrm{g})$ & $571,01 \pm 99,0$ \\
Peso pulpa $(\mathrm{g})$ & $374,54 \pm 86,4$ \\
Peso cáscara $(\mathrm{g})$ & $174,26 \pm 62,2$ \\
\hline
\end{tabular}

(Media \pm desviación estándar).

\subsection{Caracterización fisicoquímica de tres variedades de pitahaya}

En la Tabla 2 se presentan las características fisicoquímicas de las tres variedades de pitahaya. El valor de $\mathrm{pH}$ de la pitahaya roja es menor que el de las otras dos variedades. Sin embargo, no existe diferencia significativa $(p>0,05)$, entre estos valores. Los valores de $\mathrm{pH}$ son similares con los reportados para otras cactáceas tales como la pitaya y la tuna (Ochoa-Velasco y Guerrero-Beltrán, 2012; Ochoa y Guerrero, 2012). Por otra parte, la cantidad de sólidos solubles totales en la pitahaya rosa fue mayor al de las otras variedades, aunque no existió diferencia significativa $(p>0,05)$ entre las diferentes variedades. Esquivel et al. (2007a) reportaron valores de 7,5 a $13 \%$ en diferentes variedades de pitahaya, destacándose la glucosa y fructosa como 
los principales sólidos solubles totales (Esquivel y Araya-Quesada, 2012). La acidez más baja lo presentó la pitahaya de pulpa roja, seguido de la pitahaya de pulpa blanca y rosa con valores de 0,3, 0,6 y $0,7 \%$, respectivamente; siendo el ácido málico el principal ácido orgánico con concentraciones que van desde 6,08 a 8,20 $\mathrm{g} / \mathrm{L}$ en los genotipos de pitahaya de Costa Rica (Stintzing et al., 2003; Esquivel et al., 2007b). En la misma Tabla se reportan los parámetros de color $L, a$ y $b$ de las tres variedades de pitahaya (jugo). El valor de $L$ en la pitahaya de pulpa roja se encuentra a la mitad del parámetro de luminosidad (color gris). El parámetro a tiene un valor positivo (rojo) y el $b$ es negativo (azul); lo cual coincide con el modelo de colores $L, a$ y $b$, ya que el color de la pulpa se encuentra en el rango rojo. Por otra parte, para la pitahaya de pulpa rosa y blanca el parámetro de $L$ es más cercano al blanco y los parámetros de $a$ y $b$ se encuentran ligeramente declinados al lado del rojo y amarillo, respectivamente. La pitahaya rosa y blanca presentan una coloración similar al naranja bermellón y al amarillo ámbar, respectivamente.

\subsection{Evaluación del contenido de compuestos fenólicos y capacidad antioxidante de tres variedades de pitahaya}

Las frutas y hortalizas se caracterizan por el alto contenido de compuestos con capacidad antioxidante; compuestos tales como los fenoles, pigmentos y vitaminas contribuyen a retardar los daños produ- cidos a nivel del sistema nervioso central, como consecuencia del envejecimiento de las células (Bickford et al., 1997; Kuskoski et al., 2004). En la Figura 2 se presenta el contenido de compuestos fenólicos de los jugos frescos y fermentados de las tres variedades de pitahaya. El contenido de compuestos fenólicos es estadísticamente mayor $(\mathrm{p}<0,05)$ en la pitahaya de pulpa roja $(45,3 \pm 4,3 \mathrm{mg}$ de ácido gálico/100 mL de muestra), seguido de la pitahaya de pulpa rosa y blanca con valores de $32,1 \pm 0,5$ y 24,6 $\pm 0,9 \mathrm{mg}$ ácido gálico/100 $\mathrm{mL}$ de jugo, respectivamente. Probablemente el mayor contenido de compuestos fenólicos en la pitahaya de pulpa roja es debido a la presencia de pigmentos tales como las betalainas, responsables de los colores rojos (betaninas) y amarillos (betaxantinas) (Slawomir y Mizrahi, 2002). Nurliyana et al. (2010) informaron valores de compuestos fenólicos de 19,72 y 3,75 mg de ácido gálico/100 g de pulpa en pitahaya de la variedad $H$. polyrhizus y $H$. undatus, respectivamente. Por otra parte, el contenido de compuestos fenólicos en la pitahaya de pulpa roja es superior a lo reportado para otras cactáceas de coloración roja tales como la tuna y la pitaya con valores de 42 y $39 \mathrm{mg}$ de ácido gálico/ $100 \mathrm{~g}$ de muestra, respectivamente (Ochoa y Guerrero, 2012; Ochoa-Velasco y Guerrero-Beltrán, 2012). En la Figura 3 se presenta la capacidad antioxidante para los jugos frescos y fermentados de las tres variedades de pitahaya. Se observa la misma tendencia que se presentó en el contenido de compuestos fenólicos.

Tabla 2

Caracterización fisicoquímica de tres variedades de pitahaya ${ }^{\mathrm{a}}$

\begin{tabular}{lcccccc}
\hline Variedad & $\mathrm{pH}$ & $\mathrm{SST}(\%)$ & $\mathrm{AT}^{\mathrm{b}}$ & $\mathrm{L}$ & $\mathrm{a}$ & $\mathrm{b}$ \\
\hline Pitahaya roja & $5,51 \pm 0,4^{\mathrm{a}}$ & $9,03 \pm 1,8^{\mathrm{b}}$ & $0,3 \pm 0,01^{\mathrm{b}}$ & $51,35 \pm 0,7^{\mathrm{b}}$ & $74,57 \pm 0,4^{\mathrm{a}}$ & $-1,34 \pm 0,5^{\mathrm{c}}$ \\
Pitahaya rosa & $5,72 \pm 0,6^{\mathrm{a}}$ & $13,90 \pm 1,4^{\mathrm{a}}$ & $0,7 \pm 0,01^{\mathrm{a}}$ & $89,20 \pm 0,1^{\mathrm{a}}$ & $3,45 \pm 0,2^{\mathrm{b}}$ & $7,55 \pm 0,1^{\mathrm{a}}$ \\
Pitahaya blanca & $6,00 \pm 0,7^{\mathrm{a}}$ & $10,43 \pm 0,3^{\mathrm{b}}$ & $0,6 \pm 0,02^{\mathrm{a}}$ & $88,94 \pm 0,5^{\mathrm{a}}$ & $3,84 \pm 0,5^{\mathrm{b}}$ & $5,65 \pm 0,2^{\mathrm{b}}$ \\
\hline
\end{tabular}

${ }^{\mathrm{a}}$ Valores en columnas con letras diferentes para las mismas características son estadísticamente diferentes $(\mathrm{p}<$ 0,05). ${ }^{\mathrm{b}}$ Porcentaje de Acidez Titulable como ácido málico (AA).

(Media \pm desviación estándar). 
Se observa una mayor actividad antioxidante en la pulpa de pitahaya de coloración roja $(160,8 \pm 0,8 \mathrm{mg}$ de Trolox/100 $\mathrm{mL}$ de muestra), seguida de la rosa $(124,5 \pm 0,7 \mathrm{mg}$ de Trolox/100 mL de muestra) y la blanca $(58,9 \pm 2,3 \mathrm{mg}$ de Trolox/100 $\mathrm{mL}$ de muestra). Esquivel et al. (2007a) informaron que las betalainas contienen estructuras de compuestos fenólicos y no fenólicos que son los responsables de la mayor capacidad antioxidante en jugo de pitahaya, mientras que una menor cantidad de capacidad antioxidante es aportada por otros compuestos no betalainicos. Nurliyana et al. (2010) informaron que la capacidad antioxidante cuantificado por el método DPPH en pitahaya de pulpa roja (Hylocereus polyrhizus) fue mayor $(27,45 \pm 5,03 \%)$ que la reportada para pitahaya de pulpa blanca (Hylocereus undatus) con $16,56 \pm 2,96 \%$.

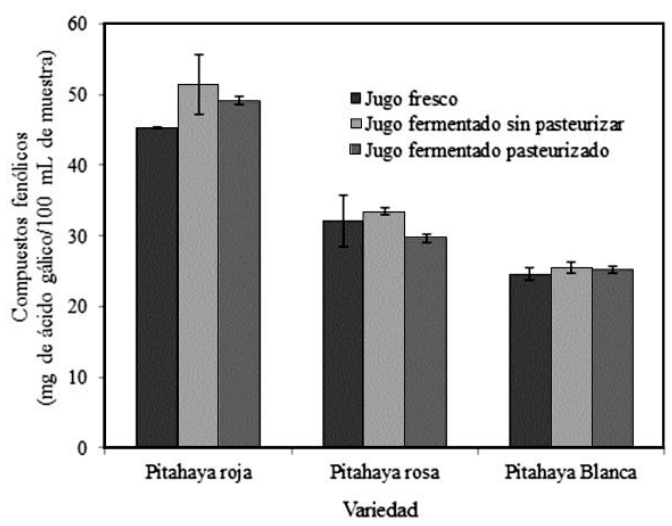

Figura 2. Contenido de compuestos fenólicos en jugo fresco y fermentado de pitahaya.

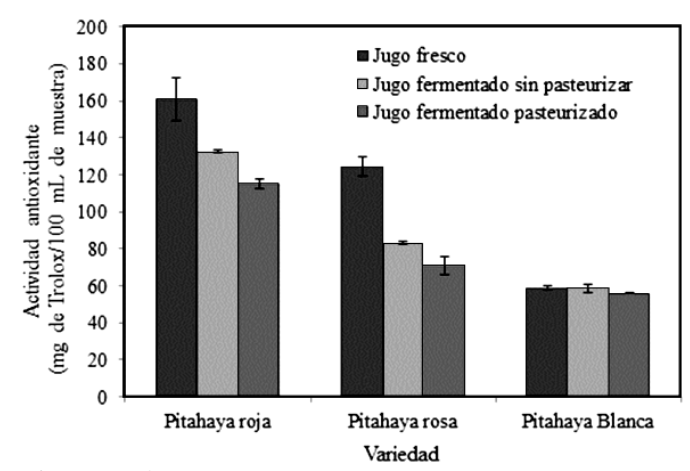

Figura 3. Capacidad antioxidante en jugo fresco y fermentado de pitahaya.

\subsection{Caracterización fisicoquímica de jugo fermentado de pitahaya}

Las características fisicoquímicas de los diferentes jugos fermentados de pitahaya se reportan en la Tabla 3. Se observa que la pasteurización no afectó el $\mathrm{pH}$, los sólidos solubles totales y la acidez titulable de los diferentes jugos fermentados. $\mathrm{El} \mathrm{pH}$ del jugo fermentado de pitahaya de pulpa blanca fue mayor al de los otros dos (frutos de pulpa rosa y roja); probablemente se debe a que el $\mathrm{pH}$ del jugo de pulpa blanca fue ligeramente mayor $(6,00)$ al de los jugos de pulpa rosa y roja $(5,72$ y 5,51 , respectivamente). $\mathrm{El} \mathrm{pH}$ es de suma importancia en los vinos, debido a que crea un equilibrio con las antocianinas (presentan diferentes formas químicas de acuerdo al $\mathrm{pH}$ ), para mantener el color del vino. Los vinos que presentan una coloración roja se debe a sus valores muy bajos de $\mathrm{pH}$; pero al momento que el $\mathrm{pH}$ aumenta, este pasa a una base de color violáceo (Peña, 2006). Fernández et al. (2009) reportaron valores de $\mathrm{pH}$ de 3,25 \pm 0,03 y $3,48 \pm 0,0$ para vino blanco y tinto, respectivamente, estos valores se encuentran ligeramente por debajo a los resultados obtenidos en el jugo fermentado de pitahaya.

Por otra parte, el menor contenido de sólidos solubles totales lo presentó el jugo fermentado de pitahaya de pulpa roja, con respecto a los de pulpa rosa y blanca. El contenido inicial de sólidos solubles totales es importante debido a que es el sustrato principal que utilizan las levaduras para la producción de alcohol (Ramírez-Mora et al., 2005). La concentración de azúcares es crítica para el desarrollo de las levaduras durante la fermentación, la principal levadura del vino (Saccharomyces cerevisiae) se alimenta de la glucosa y la fructuosa; debido a que representan más del $90 \%$ de la materia soluble (Jorgensen, 1990). Los valores obtenidos en este estudio de sólidos solubles totales son similares a los obtenidos por Ruíz (2011) en vino de arándano, quien reportó valores de $9,52-13,72 \%$. 
Tabla 3

Caracterización fisicoquímica de tres vinos de pitahaya (Hylocereus undatus) ${ }^{\mathrm{a}}$.

\begin{tabular}{|c|c|c|c|c|c|c|}
\hline \multirow[b]{2}{*}{ Variedad } & \multicolumn{2}{|c|}{$\mathrm{pH}$} & \multicolumn{2}{|c|}{ SST (\%) } & \multicolumn{2}{|c|}{$\mathrm{AT}(\% \mathrm{AM})$} \\
\hline & $\mathrm{s} / \mathrm{p}^{*}$ & $\mathrm{p}^{+}$ & $\mathrm{s} / \mathrm{p}$ & $\mathrm{p}$ & $\mathrm{s} / \mathrm{p}$ & $\mathrm{p}$ \\
\hline Pitahaya roja & $3,65 \pm 0,01^{\mathrm{a}}$ & $3,68 \pm 0,01^{\mathrm{a}}$ & $7,77 \pm 1,24^{\mathrm{a}}$ & $8,17 \pm 0,90^{\mathrm{a}}$ & $1,00 \pm 0,1^{\mathrm{a}}$ & $0,82 \pm 0,1^{\mathrm{a}}$ \\
\hline Pitahaya rosa & $3,67 \pm 0,02^{\mathrm{a}}$ & $3,68 \pm 0,02^{\mathrm{a}}$ & $8,75 \pm 0,10^{\mathrm{a}}$ & $9,02 \pm 0,71^{\mathrm{a}}$ & $0,91 \pm 0,1^{\mathrm{a}}$ & $0,81 \pm 0,2^{\mathrm{a}}$ \\
\hline Pitahaya blanca & $3,85 \pm 0,12^{\mathrm{a}}$ & $3,74 \pm 0,12^{\mathrm{a}}$ & $10,30 \pm 0,08^{\mathrm{a}}$ & $10,43 \pm 0,05^{\mathrm{a}}$ & $0,66 \pm 0,0^{\mathrm{a}}$ & $0,65 \pm 0,0^{\mathrm{a}}$ \\
\hline
\end{tabular}

${ }^{a}$ Valores en filas de las mismas características con letras diferentes son estadísticamente diferentes $(\mathrm{p}<0,05)$.

*Jugo fermentado sin pasteurizar (s/p). ${ }^{+} J u g o$ fermentado pasteurizado (p). (Media \pm desviación estándar).

Otro parámetro de calidad en un vino es su acidez, ya que ésta determina características sensoriales tales como el aroma debido a la esterificación de alcoholes (Girard, 2003). En la Tabla 3 se presenta la acidez titulable de los diferentes jugos fermentados de pitahaya. Se observa que la acidez titulable es mayor en el jugo fermentado de pitahaya roja, comparado con el de pitahaya blanca y rosa. Fernández et al. (2009) informó que los vinos tintos presentan mayor acidez que los vinos blancos, lo cual se encuentra en función de las propiedades intrínsecas de los vinos tintos. Probablemente el incremento de la acidez titulable se deba al valor que presentó el jugo antes de la fermentación. Por otra parte, la pasteurización disminuyó ligeramente la acidez titulable aunque no de manera significativa $(\mathrm{p}>0,05)$. Fernández et al. (2009) establecieron que los límites de acidez titulable para que un vino no sea considerado vinagre es $1,5 \%(0,15 \mathrm{~g} / \mathrm{L}) \mathrm{de}$ acidez titulable, expresado como ácido acético.

En la Tabla 4 se presentan los parámetros de color $L, a$ y $b$ en los diferentes jugos fermentados de pitahaya. Se puede observar que la pasteurización provocó diferentes variaciones en los parámetros de color del jugo fermentado. En el parámetro $L$ (luminosidad) se observó un incremento en el jugo fermentado de pitahaya roja y una disminución (oscurecimiento) en los otros jugos fermentados; no obstante no se observó diferencia significativa $(\mathrm{p}>0,05)$ por la pasteurización. El valor de $a$ en el jugo fermentado de pitahaya roja y blanca no varió significativamente $(\mathrm{p}>0,05)$, mientras que en el jugo fermentado de pitahaya rosa se observó un incremento ligero pero significativo $(\mathrm{p}<0.05)$. El parámetro de color $b$ se incrementó significativamente en todos los jugos fermentado de pitahaya, lo cual hace pensar que se aumentó la coloración amarilla. Morata et al. (2007) informaron que las altas temperaturas de pasteurización favorecen el oscurecimiento y cambio de color de vinos. Sin embargo, los cambios no son perceptibles al ojo humano.

\section{Tabla 4}

Evaluación de color de jugo fermentado de pitahaya ${ }^{\mathrm{a}}$.

\begin{tabular}{lcrr}
\hline Variedad & Color & $\begin{array}{c}\text { Sin } \\
\text { pasteurizar }\end{array}$ & Pasteurizado \\
\hline Pitahaya & $L$ & $64,14 \pm 0,66^{\mathrm{a}}$ & $64,81 \pm 0,66^{\mathrm{a}}$ \\
roja & $a$ & $53,95 \pm 0,96^{\mathrm{a}}$ & $51,04 \pm 0,93^{\mathrm{a}}$ \\
& $b$ & $-12,74 \pm 1,7^{\mathrm{b}}$ & $-10,56 \pm 1,7^{\mathrm{a}}$ \\
\hline Pitahaya & $L$ & $90,40 \pm 0,19^{\mathrm{a}}$ & $89,20 \pm 0,19^{\mathrm{a}}$ \\
rosa & $a$ & $2,65 \pm 0,21^{\mathrm{b}}$ & $3,45 \pm 0,21^{\mathrm{a}}$ \\
\hline \multirow{2}{*}{ Pitahaya } & $b$ & $6,27 \pm 0,07^{\mathrm{a}}$ & $7,55 \pm 0,07^{\mathrm{b}}$ \\
blanca & $a$ & $95,10 \pm 0,36^{\mathrm{a}}$ & $94,65 \pm 0,36^{\mathrm{a}}$ \\
& $b$ & $-2,55 \pm 0,20^{\mathrm{a}}$ & $-2,17 \pm 0,20^{\mathrm{a}}$ \\
\hline
\end{tabular}

a Valores en filas con letras diferentes son estadísticamente diferentes $(\mathrm{p}<0,05)$. (Media \pm desviación estándar).

\subsection{Evaluación del contenido de compuestos fenólicos y capacidad antioxidante de jugos fermentados de pitahaya}

Los vinos se caracterizan por el alto contenido de compuestos fenólicos obtenidos a partir de la piel, semillas y pulpa de uva que son mezclados en el proceso de vinificación (McDonald et al., 1998). Los principales compuestos fenólicos en vino tinto son flavonas, 
quercentina, miricetina y antocianinas (Burns et al., 2001). En la Figura 2 se presenta el contenido de compuestos fenólicos de jugo fermentado de pitahaya. El contenido de compuestos fenólicos se incrementó significativamente $(\mathrm{p}<0,05)$ en el jugo fermentado de pitahaya de pulpa roja comparado con el jugo (mosto) inicial, estas mismas tendencias de incremento de los compuestos fenólicos fueron encontradas en cuatro diferentes vinos tintos (Burns et al., 2001). González-Neves et al. (2003) informaron que el tiempo de maceración (7 días) del vino tinto incrementó de manera importante el contenido de compuestos fenólicos, sin embargo largos tiempos de maceración disminuyen el contenido de compuestos fenólicos. El producto obtenido a partir de la fermentación de jugo de pitahaya de pulpa roja presentó el mayor contenido de compuestos fenólicos (51 mg ácido gálico/100 mL de muestra), seguido del de pulpa rosa $(33 \mathrm{mg}$ de ácido gálico/100 mL de muestra) y pulpa blanca $(25 \mathrm{mg}$ de ácido gálico/100 mL de muestra). Por otra parte, la pasteurización disminuyó el contenido de compuestos fenólicos, aunque no de manera significativa ( $p>$ 0,05 ) en el jugo fermentado de pulpa roja.

El contenido de compuestos fenólicos es menor a lo reportado para vino tinto. Barreiro et al. (2006) reportaron valores de compuestos fenólicos de $165,1 \mathrm{mg}$ de ácido gálico/100 mL de vino tinto de uva variedad Tannat. Sin embargo, el contenido de compuestos fenólicos en vino se encuentra en función de diferentes factores tales la variedad de uva, las condiciones y tiempo de almacenamiento entre otras (Burns et al., 2001).

En la Figura 3 se presentan los resultados de la capacidad antioxidante de los tres jugos fermentados de pitahaya. Contrariamente a lo ocurrido con los compuestos fenólicos el proceso de fermentación disminuyó de manera significativa $(\mathrm{p}<0,05)$ la capacidad antioxidante en el jugo fermentado de pitahaya de pulpa roja y rosa. Esto probable se deba a una disminución de los compuestos betalainicos no fenólicos presentes en el jugo fermentado de pitahaya de pulpa roja y rosa. Como era de esperarse la capacidad antioxidante fue mayor significativamente $(p<0,05)$ en el jugo fermentado de pitahaya roja, seguido de la de pulpa rosa y la de pulpa blanca. Aunado a lo anterior se observa que la pasteurización disminuyó de manera significativa $(\mathrm{p}<0,05)$ la capacidad antioxidante de todos los jugos fermentados.

Es importante destacar que la capacidad antioxidante de los tres jugos fermentados de pitahaya son superiores a los reportados en un estudio realizado a más de 99 vinos españoles de distintas variedades de uvas y clasificados en vinos tintos, rosados $\mathrm{y}$ blancos con valores promedio de 114,9, 23,3 y 12,9 mg Trolox/100 mL de muestra, respectivamente (Rodríguez y García, 2005).

\subsection{Grado alcohólico de jugo fermentado de pitahaya}

En la Tabla 5 se presenta el porcentaje de alcohol o grado alcohólico de jugo fermentado de pitahaya. Se puede observar que el contenido de alcohol en los diferentes jugos fermentados de pitahaya se encuentra muy por debajo de los obtenidos en otros fermentados, como los de uva y arándano, con valores de 12,26$12,39 \%$ y $11,95-12,91 \%$, respectivamente (Fernández et al., 2009; Ruíz, 2011). Mientras que Ferreyra et al. (2009) informaron que el contenido alcohólico en vino de naranja fue de $6-10 \%$. Probablemente el menor grado alcohólico en los jugos fermentados de pitahaya se deba al contenido de sólidos solubles totales $(15 \%)$ al iniciar la fermentación. No obstante, los porcentajes alcohólicos encontrados en este estudio se encuentran dentro de lo establecido por NOM-142SSA1-1995 establecida para bebidas carbonatadas y sidra (3-8 \% de alcohol). Se observa que la pasteurización redujo el contenido alcohólico de manera importante 
en los distintos jugos fermentados de pitahaya, esto se debe probablemente a que el etanol se volatiliza con facilidad y se evapora fácilmente a las temperaturas de pasteurización (Nisperos-Carriedo y Shaw, 1990).

\section{Tabla 5}

Contenido de alcohol de jugo fermentado de pitahaya ${ }^{\mathrm{a}}$.

\begin{tabular}{lcc}
\hline Variedad & $\begin{array}{c}\text { Sin } \\
\text { pasteurizar } \\
(\%)\end{array}$ & $\begin{array}{c}\text { Pasteurizado } \\
(\%)\end{array}$ \\
\hline Pitahaya roja & $3,9 \pm 0,1^{\mathrm{a}}$ & $3,0 \pm 0,1^{\mathrm{b}}$ \\
Pitahaya rosa & $3,0 \pm 0,2^{\mathrm{a}}$ & $2,9 \pm 0,1^{\mathrm{a}}$ \\
Pitahaya blanca & $2,9 \pm 0,2^{\mathrm{a}}$ & $2,7 \pm 0,3^{\mathrm{a}}$ \\
\hline
\end{tabular}

a Valores en filas con letras diferentes son estadísticamente diferentes $(\mathrm{p}<0.05)$. (Media \pm desviación estándar).

\subsection{Conteo microbiológico de los jugos fermentados de pitahaya}

En la Tabla 6 se presentan los resultados de bacterias mesófilas aerobias, mohos y levaduras en los jugos fermentados de pitahaya. Los resultados obtenidos indican que la pasteurización eliminó el crecimiento de ambos tipos de microorganismos, comparado con los jugos fermentados no pasteurizados; ya que se puede observar presencia microbiana de bacterias mesófilas aerobias en jugo fermentado de pulpa roja y rosa; así como también crecimiento de mohos y levaduras en los tres jugos fermentados de pitahaya (sin pasteurizar). En el caso del vino la OIV (Organización Mundial de la viña y el vino) no especifica un nivel máximo de carga microbiana, la única condición es mantener una apariencia clara en la botella, lo que supone una carga microbiana menor a $10^{4} \quad \mathrm{UFC} / \mathrm{mL}$ (vinos blancos) para organismos productores de sedimentos flotantes, y menores de $10^{2} \mathrm{UFC} / \mathrm{mL}$ para microorganismos floculantes (Loureiro $\mathrm{y}$ Malfeiti-Ferreira, 2003). Generalmente, los vinos dulces tienen mayor restricción de niveles aceptables, debido a que el crecimiento de la levadura es mayor o más evidente que en vinos secos; por lo que los vinos pasteurizados tienen un recuento de
$\mathrm{UFC} / \mathrm{mL}$ aceptable (Chatonnet et al., 1992).

Tabla 6

Análisis microbiológico los vinos de pitahaya ${ }^{\mathrm{a}}$

\begin{tabular}{|c|c|c|c|c|}
\hline \multirow[b]{2}{*}{ Variedad } & \multicolumn{2}{|c|}{$\begin{array}{c}\text { BMA } \\
\text { (UFC/mL) } \\
\end{array}$} & \multicolumn{2}{|c|}{$\begin{array}{c}\text { M y L } \\
\text { (UFC/mL) }\end{array}$} \\
\hline & $\begin{array}{c}\text { Sin } \\
\text { Pasteuri- } \\
\text { zar }\end{array}$ & $\begin{array}{c}\text { Pas- } \\
\text { teuri- } \\
\text { zado } \\
\end{array}$ & $\begin{array}{c}\text { Sin } \\
\text { Pasteuri- } \\
\text { zar }\end{array}$ & $\begin{array}{l}\text { Pas- } \\
\text { teuri- } \\
\text { zado }\end{array}$ \\
\hline $\begin{array}{l}\text { Pitahaya } \\
\text { roja }\end{array}$ & $\begin{array}{r}165 \\
\pm 39^{\mathrm{a}} \\
\end{array}$ & $<10^{\mathrm{b}}$ & $\begin{array}{r}1540 \\
\pm 368^{a} \\
\end{array}$ & $<10^{b}$ \\
\hline $\begin{array}{l}\text { Pitahaya } \\
\text { rosa }\end{array}$ & $\begin{array}{c}57 \\
\pm 7^{\mathrm{a}}\end{array}$ & $<10^{\mathrm{b}}$ & $\begin{array}{r}1010 \\
\pm 200^{\mathrm{a}}\end{array}$ & $<10^{\mathrm{b}}$ \\
\hline $\begin{array}{l}\text { Pitahaya } \\
\text { blanca }\end{array}$ & $\begin{array}{r}48 \\
\pm 2^{\mathrm{a}} \\
\end{array}$ & $<10^{\mathrm{b}}$ & $\begin{array}{r}1580 \\
\pm 311^{\mathrm{a}} \\
\end{array}$ & $<10^{\mathrm{b}}$ \\
\hline
\end{tabular}

${ }^{a}$ Valores en filas de los mismos microorganismos con letras diferentes son estadísticamente diferentes ( $\mathrm{p}<0,05)$. (Media \pm desviación estándar).

\section{Conclusiones}

A partir de los resultados obtenidos se concluye que la pitahaya es una cactácea con alto contenido de sólidos solubles totales $(9,03-13,90)$ y alto $\mathrm{pH}(5,51-6,00)$ que dificultan su conservación en fresco debido a las condiciones ideales para el desarrollo microbiano. Por otra parte, es una fruta con un alto contenido de compuestos fenólicos $(45,31 \mathrm{mg}$ de ácido gálico/100 ml de jugo) y capacidad antioxidante (160,84 $\mathrm{mg}$ de Trolox/100 $\mathrm{mL}$ de jugo) (pitahaya de pulpa roja), incluso superiores a los de otras cactáceas rojas como la tuna y la pitaya. La elaboración de jugo fermentado a partir de tres variedades de pitahaya es de fácil y rápida elaboración, obteniéndose productos con un alto contenido en compuestos fenólicos y antioxidantes. Es importante recalcar que la pasteurización disminuye factores de calidad tales como los compuestos fenólicos, capacidad antioxidante y grado alcohólico. Sin embargo, la pasteurización es importante para disminuir la carga microbiana de los jugos. Aunque los parámetros de color se incrementaron, este cambio no fue visible a la vista humana. La producción de jugo fermentado a partir de pitahaya puede ser una alternativa viable para los productores de comercializar la fruta. 


\section{Referencias bibliográficas}

AOAC. 2000. Official Methods of Analysis. 14 a edition. Association of Official Analytical Chemists, Inc. Washington D.C., EUA.

Barreiro, L.; Charamelo, D.; González-Neves. 2006. Perfil antociánico y composición fenólica de vinos Tannat elaborados con adición de enzimas pectolíticas. Revista de la Facultad de Ciencias Agrarias Universidad Nacional de Cuyo. 2:9-18.

Betancourt, B.; Toro, J.C.; Mosquera, H.A.; Castellanos, J.C.; Martínez, R.M.; Aguilera, A.A.; Perdomo, L.E.; Franco, M.A. 2010. Agenda prospectiva de investigación y desarrollo tecnológico para la cadena productiva de la pitaya amarilla en fresco en el Valle de Cauca. Ministerio de Agricultura y Desarrollo Rural. Bogotá, Colombia.

Bickford, P.C.; Chadman, K.; Taglialatea, G.; ShukittHale, B.; Prior, R.L.; Cao. C.; Joseph, J.A. 1997. Dietary strawberry supplementation protects against the age-accelerated CNS effects of oxidative stress. Federation of American Societies for Experimental Biology. 11: A176.

Brown, C.M.; Campbell, I.; Priest, F.G. 1989. Introducción a la Biotecnología. Editorial Acribia, S.A. Zaragoza, España.

Burns, J.; Gardner, P.; Matthews, D.; Duthie, G.; Lean, M.; Crozier, A. 2001. Extraction of phenolics and changes in antioxidant activity of red wines during vinification. Journal of the Science and Food Chemistry. 49: 5797 5808 .

Castillo-Martínez, R.; Livera-Muñoz, M.; MárquezGuzmán, G.J. 2005. Caracterización morfológica y compatibilidad sexual de cinco genotipos de pitahaya (Hylocereus undatus). Agrociencia. 39:183-194.

Castillo, R. 2006. Aprovechamiento de la pitahaya: bondades y problemáticas. Caos Conciencia. 1:13-18.

Cerdas, M.M.; Montero, M.; Díaz, E. 2006. Manual de manejo pre y poscosecha de aguacate (Persea americana). Ministerio de Agricultura y ganadería. Centro de investigaciones agronómicas. Fundación para el Fomento y Promoción de la Investigación y Transferencia de Tecnología Agropecuaria de Costa Rica. San José, Costa Rica.

Chatonnet, P.; Barbe, C.; Canal-Llauberes, R.M.; Dubourdieu, D.; Boidron, J.N. 1992. Incidences de certaines preparations pectolytiques sur la teneur en phenols volatils des viris blancs. Journal International des Sciences de la Vigne et du Vin. 26:253-269.

Corrales-García, J.; Canche-Canche, E. 2008. Physical and Physiological changes in low temperature-stored pitahaya fruit (Hylocereus undatus). Journal of the Professional Association for Cactus Development 10:108-119.

Esquivel, P.; Araya-Quesada, Y. 2012. Características del fruto de la pitahaya (Hylocereus sp.) y su potencial de uso en la industria alimentaria. Revista Venezolana de Ciencia y Tecnología de Alimentos. 3: 113-129.

Esquivel, P.; Stintzing, F.C.; Carle, R. 2007a. Fruit characteristics during growth and ripening of different Hylocereus genotypes. European Journal of Horticultural Science. 72:231-238.

Esquivel, P.; Stintzing, F.C.; Carle, R. 2007b. Comparison of morphological and chemical fruit traits from different pitaya genotypes (Hylocereus sp.) grown in Costa Rica. Journal of Applied Botany and Food Quality. 81:7-14.

EurLex. 2004. Métodos de análisis comunitarios aplicables en el sector del vino. Reglamento (CR) $n^{\circ} 128 / 2004$ de la Comisión. Disponible en: http://eurlex.europa.eu/LexUriServ/site/es/oj/2004/1_019/1_0192 0040127es00030011.pdf.

Fernández, V.; Berradre, M.; Sulbarán, B.; Ojeda de Rodríguez, G.; Peña, J. 2009. Caracterización química y contenido mineral en vinos comerciales venezolanos. Revista de la Facultad de Agronomía de la Universidad de Zulia. 26: 382-397

Ferreyra, M.M.; Schvab, M. del C.; Gerard, L.M.; Zapata, L.M.; Davies, C.V.; Hours, R.A. 2009. Fermentación alcohólica de jugo de naranja con $S$. cerevisiae. Ciencia, Docencia y Tecnología. 39: 143-158.

Gao, X.; Ohlander, M.; Jeppsson, N.; Bjök, L.; Trajkovsky, V. 2000. Changes in antioxidants effects and their relationships to phytonutrients in fruits of sea during maduration buckthorn (Hippophae rhamnoides). Journal of Agriculture and Food Chemistry. 48:14854190.

Girard, G. 2003. Bases Científicas y Tecnológicas de la Enología. Editorial Acribia, S.A. Zaragoza, España. pp: $238-240$.

González-Neves, G.; Balado, J.; Barreiro, L.; Bochicchio, R.; Gatto, G.; Gil, G.; Tessore, A.; Ferrer, M. 2003. Efecto de algunas prácticas de manejo del viñedo y de la vinificación en la composición fenólica y el color de los vinos tintos. X Congresso Brasileiro de Viticultura e Enologia. Bento Goncalves, Brasil. 3-5 de Diciembre.

Jorgensen, A. 1990. Microbiología de las fermentaciones industriales. Séptima edición. Editorial Acribia, S.A. Zaragoza, España. pp: 22-25.

Kuskoski, M.; Asuero, A.; Parrilla, M.; Troncoso, A.; Fett, R. 2004. Actividad antioxidante de pigmentos antociánicos. Ciencia y Tecnología de Alimentos 24: 691-693.

Legaria, J.P.; Alvarado, M.E.; Gaspar, R. 2005. Diversidad genética en pitahaya (Hylocereus undatus Haworth. Britton y Rose). Revista Fitotecnia Mexicana. 28: 179185

Le, V.T.; Nguyen, N.; Nguyen, D.D.; Dang, K.T.; Nguyen, T.N.C.; Dang, M.V.H.; Chau, N.H.; Trink, N.L. 2000. Quality assurance system for dragon fruit. ACIAR Proceedings. 100:101-114.

Loureiro, V.; Malfeito-Ferreira, M. 2003. Spoilage yeasts in the wine industry. International Journal of Food Microbiology. 86: 23-50.

McDonald, M. S.; Hughes, M.; Burns, J.; Lean, M. E. J.; Matthews, D.; Crozier, A. 1998. Survey of the free and conjugated myricetin and quercetin content of red wines of different geographical origins. Journal of Agriculture and Food Chemistry. 46: 368-375.

Morata, A.; Benito, S.; Palomero, F.; Calderón, F.; SuárezLepe, J.A. 2007. Ecología y prevención de Brettanomyces/Dekkera durante la elaboración y crianza de vinos tintos. Revista Enólogos. 50:1-12.

Nisperos-Carriedo, M.O.; Shaw, P.E. 1990. Comparison of volatile flavor components in fresh and processed orange juices. Journal of Food Agriculture and Food Chemistry. 38:1048-1052.

Norma Oficial Mexicana NOM-092-SSA1-1994. Bienes y servicios. Método para la cuenta de bacterias aerobias en placa. Disponible en: http://www.salud.gob.m x/unidades/cdi/nom/092ssa14.html

Norma Oficial Mexicana NOM-111-SSA1-1994. Bienes y servicios. Método para la cuenta de mohos y levaduras en alimentos. Disponible en: http://www.salud. salud.gob.mx/unidades/cdi/nom/111ssa14.html

Norma Oficial Mexicana NOM-142-SSA1-1995. Bienes y servicios. Bebidas Alcoholicas. Especificaciones Sanitarias. Etiquetado Sanitario y Comercial. 
Disponible en: http://www.salud.gob.mx/unidades/ cdi/nom/142ssa15.html.

Nurliyana, R.; Syed-Zahir, I.; Mustapha-Suleiman, K.; Aisyah, M.R.; Kamarul Rahim, K. 2010. Antioxidant study of pulps and peels of dragon fruits: a comparative study. International Food Research Journal. 17:367-375.

Ochoa, C.E.; Guerrero, J.A. 2012. Efecto del almacenamiento a diferentes temperaturas sobre la calidad de tuna roja (Opuntia ficus indica (L.) Miller). Información Tecnológica. 23:117-128.

Ochoa-Velasco, C.E.; Guerrero-Beltrán, J.A. 2012. Ultraviolet-C light effect on pitaya (Stenocereus griseus) juice. Journal of Food Research. 1:60-70.

Peña, A. 2006. El color de los vinos. Revista Vendimia. Enero-Febrero. 24-26.

Ramírez-Mora, E.; Martínez, R.A.; Fernández-Montes, M.R. 2005. Efecto de la concentración de azúcar y la cepa de levadura en la calidad de sidra espumosa. Brazilian Journal of Food Technology. 5:89-95.

Raveh, E.; Weiss, J.; Nerd, A.; Mizrahi, Y. 1993. Pitayas (genus Hylocereus): A new fruit crop for the Negev Desert of Israel. En New Crops. Janick, J., Simon, J.E. Eds; Wiley. New York. pp: 491-495.
Rodríguez, R.; García, E. 2005. Actividad antioxidante y composición fenólica en vinos de Castilla-La mancha. Alimentaria. Revista de tecnología de higiene de los alimentos. 362:128-133.

Ruíz, H. 2011. Desarrollo de un vino de mortiño (arándanos) en la corporación grupo salinas de Ecuador. Universidad Pública de Navarra. Tesis de ingeniería en agronomía. Pamplona, España.

SIAP. 2011. Cierre de la producción agrícola por cultivo. Disponible en: http://www.siap.gob.mx/index.php? option $=$ com_wrapper $\&$ view=wrapper $\&$ Itemid $=350$.

Slawomir, W.; Mizrahi, Y. 2002. Fruit flesh betacyanin pigments in Hylocerus Cacti. Journal of Agricultural and Food Chemistry. 50: 6086-6089.

Stintzing, C.; Schieber, A.; Reinhold, C. 2003. Evaluation of colour properties and chemical quality parameters of cactus juices. European Food Research and Technology. 216:303-311.

Wybraniec, S.; Mizrahi, Y. 2002. Fruit flesh betacyanin pigments in Hylocereus cacti. Journal of Agricultural and Food Chemistry. 50:6086-6089. 